\title{
Estudio de la Fermentación del Mosto de Uva Italia (Vitis vinífera L) Ejecutada sin Hollejos y su Influencia en el Pisco Puro Aromático
}

RESPONSABLE: MSc. Samuel Cerro Ruiz.

RESUMEN La investigación demostró que un proceso fermentativo del mosto de uva Italia (Vitis vinífera L), ejecutado sin presencia de hollejos (orujos) y con control de temperatura, influye en las características químicas y organolépticas del pisco puro aromático.

La destilación se ejecutó según procedimientos y parámetros ya conocidos, obteniéndose un pisco con $43,6 \%$ vol. de grado alcohólico y rendimiento de $21,6 \%$

Quimicamente se demostró que los procesos fermentativos de mostos en ausencia de hollejos nos permite obtener piscos con bajos contenidos de alcoholes tóxicos como el metanol originado en la maceración-fermentación por la degradación de sustancias pécticas presentes en los hollejos y pulpa de la uva y que reportó un valor de $34,2 \mathrm{mg} / 100 \mathrm{ml} \mathrm{AA}$, considerando que la NTP $N^{2} 211.001-2006$, permite hasta $150 \mathrm{mg} / 100 \mathrm{ml} \mathrm{AA} \mathrm{para} \mathrm{este} \mathrm{tipo} \mathrm{de} \mathrm{pisco.}$

Un panel semientrenado de jueces calificó por preferencia hedónica $y$ dio un puntaje promedio de 8,187 sobre 9,0 puntos que equivale al calificativo de muybueno.

Palabras clave: pisco puro aromático Italia ; fermentación, destilación, evaluación organoléptica.
MIEMBro: $D r$ Miguel Larrea Céspedes

ABSTRACT This research has demonstrated that fermentative processes of Italia grape (Vitis vinifera L.) obtained without the presence of less (grape skins) and under controlled temperature conditions, influences on the chemical and sensorial characteristics of pisco puro aromatico.

Distillation was accomplished with the most common conditions described to obtain Pisco, achieving 43, 6\% alcoholic degrees and a yield of $21,6 \mathrm{~L}$.

Chemically was demonstrated that fermentative processes of grape juice without the presence of lees, allow the obtaining of piscos with low levels of toxic alcohols as methanol, which is originated on the maceration of the must, initiated on the degradation of peptic compounds present on the lees and grape pulp. Was reported a value of $34,2 \mathrm{mg} / 100 \mathrm{~mL} A A$, considering that NTP (Norma Tecnica Peruana) $n$ 211.011 of 2006 allows until $150 \mathrm{mg} / 100 \mathrm{~mL} \mathrm{AA}$ for this kind of pisco.

A semi-trained panel of judges qualifying by hedonic preference gave an average score of 8,19 over 9,0 points, that corresponds to a very good attribute. Keywords: pisco puro aromático Italia; fermetation, destillation, sensorial evaluation.

propiedades físico-químicas y organolépticas del pisco puro aromático.

Objetivos Especificos

1. Evaluar los principales parámetros de fermentación y destilación del mosto de uva Italia destilado en alambique de cobre sin estañar.

2. Caracterizar físico-química y organolépticamente el pisco puro aromático.

\section{HIPÓTESIS}

Es posible evaluar las condiciones y parámetros durante la fase fermentativa y verificar su influencia en las características físico-químicas y organolépticas de un pisco puro aromático de uva Italia.

\section{VARIABLES}

Variable independiente

- Retiro del hollejo antes de la fermentación del mosto.

- Medición de parámetros durante la fermentación y 
destilación.

Variable dependiente

- Características físico-químicas y organolépticas.

\section{INDICADORES}

Requisitos de la Norma Técnica Peruana 211.001: 2006. Pisco-Requisitos

\section{MATERIALESYMÉTODOS}

\section{Lugar de ejecución}

Los procesos y sus controles de fermentación, destilación y evaluación organoléptica se ejecutaron en los laboratorios de la Facultad de Ingeniería en Industrias Alimentarias de la Universidad Nacional Jorge Basadre Grohmann, (FAIA/UNJBG) en Tacna.

Los análisis físico - químicos del pisco puro fueron hechos por cromatografia de gases en los laboratorios de La Molina Calidad Total de Lima

\section{Materiales y métodos de análisis}

Fueron utilizadas uvas de la variedad Italia (Vitis vinifera $L$ ) de la irrigación Magollo, Tacna y una serie de materiales y equipos ad hoc a la investigación.

Los principales métodos de análisis estuvieron basados en los métodos oficiales de la A.O.A.C. (Amerine y Ough-1976). En métodos oficiales de la O.I.I.V (1976) y en las Norma Técnica Peruana 211.001: 2006; NTP 210.003 : 2003; NTP 211.040: 2003; NTP 211.035: 2003; NTP 210.025: 2003; NTP 211.038: 2003 ;NTP 211.041:2003;NTP 210.022: 2003

\section{RESULTADOS Y DISCUSIÓN}

\section{Materia Prima}

El control de la materia prima resultó en : -Sólidos solubles 26,4 ${ }^{\circ} \mathrm{Bx}$; - Densidad 1, $111 \mathrm{~g} / \mathrm{L}$; - Densidad 14,6 a $\mathrm{Be}$; - Acidez tartárica total $5,2 \mathrm{~g} / \mathrm{L} ;-\mathrm{pH} 4,0$

\section{Del Pie de Cuba}

El desarrollo del proceso fermentativo por 72 horas con levaduras nativas activas en el pie de cuba se dio a condiciones ambientales y se obtuvieron $0,76 \mathrm{~L}$. de mosto fermentado.

\section{Estrujado Despalillado y Retiro de los Hollejos}

Se empezó con $12,860 \mathrm{~kg}$ de uva sana, madura que fue estrujada y de la cual se retiraron los escobajos y enseguida los orujos u hollejos obteniéndose el mosto.

\section{Fermentación del Mosto de Uva Sin Hollejos y con Pie de Cuba}

A $8,4 \mathrm{~L}$ de mosto (jugo y pulpa) se adicionó el pie de cuba $(0,76 \mathrm{~L})$, homogenizándolo y luego fue dejado fermentar nueve días con controles dos veces al día de remontajes y parámetros como tiempo, temperatura (menor de $25,5^{\circ} \mathrm{C}$ ), densidad, sólidos solubles, acidez total y $\mathrm{pH}$. No se adicionó compuesto sulfurado ni levadura seleccionada.

Al vino base con densidad final de $992 \mathrm{~g} / \mathrm{L}$ le correspondió una riqueza alcohólica de $11,1 \%$ vol. 7,5 ${ }^{\circ}$ Brix y acidez total de $6,8 \mathrm{~g} / \mathrm{L}$ como ácido tartárico

\section{Descubado}

Terminada la fermentación se retiró el vino base dejando una cantidad de borras gruesas y finas depositadas en el fondo. La cantidad de vino base obtenido fue de $5,4 \mathrm{~L}$.

\section{Reposo del Vino Base}

Se dejó reposar el vino base obtenido hasta el final del dia siguiente, en que fue separado de sus borras finas,

\section{Destilación}

Se pusieron a destilar 5,4 $\mathrm{L}$ de vino base calentados a fuego directo moderado.

a) "Cabeza". Se cortó a los primeros $54 \mathrm{ml}$ de la destilación ( $1 \%$ del volumen depositado en paila), con $64,10 \%$ vol. que contienen alcoholes superiores tóxicos (isoamílico, isobutílico, propílico y butílico); compuestos aromáticos (acetaldehido, acetato de etilo, ésteres, terpenos) y saborizantes que le confieren características desagradables (picante, áspero, acre). Suelen desprenderse a temperaturas de destilación por encima de $78,4^{\circ}$.

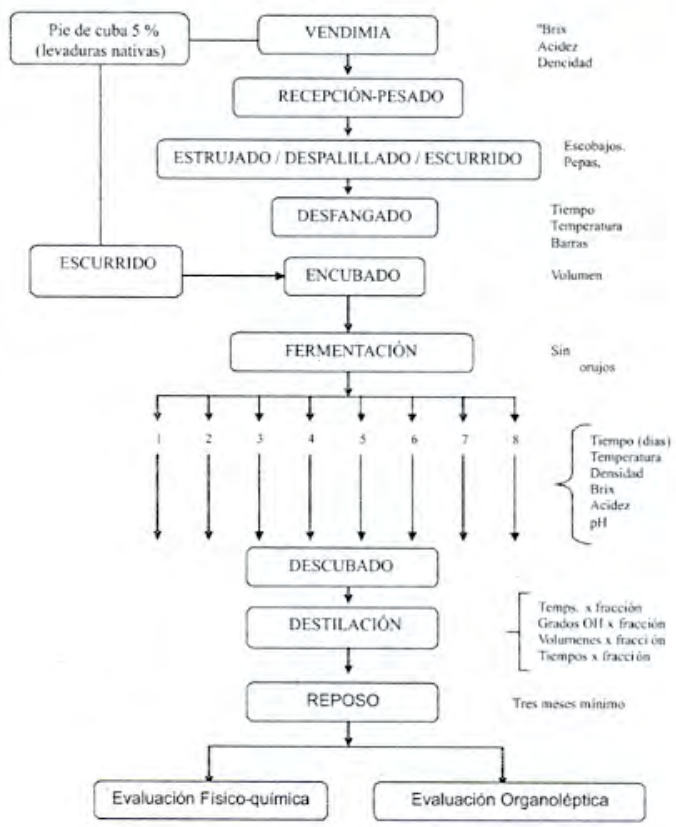

Figura $\mathrm{N}^{\circ} 01$ Flujo experimental de elaboración de pisco puro aromático Italia

b) "Cuerpo". Se obtuvieron $1100 \mathrm{ml}$ con graduación de $43,6 \%$ vol. en la mezcla hidro alcohólica que fue el grado al cual se hizo el corte entre "cuerpo"y "cola". El 
rendimiento fue de $21,56 \%$

c) "Cola". Se alcanzó un volumen de $40 \mathrm{ml}$ con un grado de $9,3 \%$ vol. que fueron apartados por ser compuestos tóxicos y nos referimos a furfural, metanol, ácido butírico que van acompañados de aromas y sabores indeseables. Junto con las cabezas forman los llamados "puchos".

\section{De La Evaluación Físico-Química del Pisco Puro Aromático}

Evaluación física

Se muestran a continuación:

Cuadro $\mathrm{N}^{\circ} 01$ Caracteristicas fisicas del pisco puro obtenido

\begin{tabular}{|l|c|}
\hline \multicolumn{1}{|c|}{ Componente físico } & \multicolumn{1}{|c|}{$\begin{array}{c}\text { Valores } \\
\text { Obtenidos }\end{array}$} \\
\hline $\begin{array}{l}\text { Grado alcohólico (\% vol.) a } \\
20 / 20^{\circ} \mathrm{C}\end{array}$ & 43,6 \\
\hline Extracto seco $(\mathrm{g} / \mathrm{L})$ a $100^{\circ} \mathrm{C}$ & 0,06 \\
\hline Densidad $(\mathrm{g} / \mathrm{L})$ a $20 / 20^{\circ} \mathrm{C}$ & 0,9428 \\
\hline pHa $20 / 20^{\circ} \mathrm{C}$ & 3,6 \\
\hline
\end{tabular}

Fuente: Elaboración propia (2008)

Evaluación química por cromatografía

La evaluación química fue realizada por cromatografia de gases en el Laboratorio de Química de La Molina Calidad Total en Lima

\section{Comparación de resultados}

Todos los valores del pisco en evaluación estuvieron dentro de los rangos especificados por la NTP $N^{0} 211.006$ : 2006, lo cual nos indica que las acciones de control en las etapas de fermentación y destilación fueron las correctas y podemos hacer unos alcances comparativos respecto de un trabajo similar desarrollado por Hatta B. y Palma J.C. (2004) pero con uva Italia del valle de Ocucaje, Ica.

Cuadro $\mathrm{N}^{\circ} 02$ Caracteristicas quimicas del pisco aromático Italia fermentado sin hollejos

\begin{tabular}{|c|c|c|c|}
\hline $\begin{array}{l}\text { Componentes velátiles y odoriferos } \\
\text { ( } \mathrm{mg} / 100 \mathrm{ml} \text { de } \mathrm{AA})\end{array}$ & $\begin{array}{l}\text { Resultados } \\
\text { Mhuestra }\end{array}$ & \multicolumn{2}{|c|}{$\begin{array}{c}\text { Requisitos } \\
\text { NTP N211.001 : } 2006\end{array}$} \\
\hline - Esteres como acetato de etilo & 12.6 & 10.0 & 330,0 \\
\hline - Forniato de etilo (1) & 0.0 & - & - \\
\hline - Acetato de etilo & 12.6 & 10.0 & 280,0 \\
\hline - Acetato de lo amibo (1) & 0.0 & - & - \\
\hline - Furfural & 0.5 & 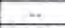 & 5.0 \\
\hline - Aldehidos como Acetaldehido & 10,7 & 3,0 & 50,0 \\
\hline - Akoholes superiores totales. & 201.4 & 60.0 & 350.0 \\
\hline - Iso propanol (2) & 0.0 & - & - \\
\hline - Pronanol (2) & 29,0 & - & - \\
\hline - Butanol (2) & 1.5 & - & - \\
\hline-1 so butanol (2) & 34.5 & - & - \\
\hline $\begin{array}{l}3 \text { metil-1 butanol/2-metil-1- } \\
\text { butanol (2) }\end{array}$ & 136.4 & - & - \\
\hline r. Acidez volátil (ac acéticon) & 0,4 & - & 200,0 \\
\hline Akohol metilico & 34.2 & 4.0 & 150.0 \\
\hline
\end{tabular}

Fuente: Elaboración propia (2008).

Notas : (1) Es posible que no estén presentes, pero de estarlos, la suma con el acetato de etilo no debe sobre pasar 330 $\mathrm{mg} / 100 \mathrm{ml}$.

(2) Deben estar presentes sin precisar exigencias de máximos y mínimos.

El tiempo de fermentación en Tacna fue de nueve dias mientras que para la referencia duró ocho días; las densidades y grado alcohólicos de los dos vinos base tuvieron valores de $992 \mathrm{~g} / \mathrm{L}$ y $11,1 \%$ vol. el de Tacna y el de su similar iqueña $995 \mathrm{~g} / \mathrm{L}$ y $11,5 \%$ vol.

En Tacna, se evaluó la acidez total en términos de ácido tartárico y en el otro caso lo midieron como acidez volátil expresado en términos de ácido acético. Los valores son diferentes.

La operación de destilación fue similar en ambos ensayos : para el corte de "cabeza" se adoptó un valor de $1 \%$ del volumen del vino base total; para el corte de "cuerpo" se tomaron como referencia en ambos casos la graduación resultante de la mezcla hidro alcohólica que en nuestra caso fue de $43,6 \%$ vol. y en la referencia iqueña fue de $40,9 \%$ vol.

En cuanto a los alcoholes superiores, la mayoría de ellos (propanol, butanol, isobutanol, isoteramílico) aparecen en los primeros minutos del destilado, o sea en la "cabeza", salvo el isopropanol que tiene punto de ebullición más elevado y aparece en la fracción "cola" de la destilación.

Para el caso de los ésteres, dos de ellos acetato de etilo y acetato de isoamilo, destilan en un porcentaje importante en la fracción "cabeza". En nuestra muestra el segundo éster no apareció. Un tercero, el formiato de etilo se va desprendiendo en la parte final de la fracción "cuerpo" y mayormente en la fracción "cola"; tampoco apareció en nuestro pisco

Respecto de los aldehídos, el acetaldehído se desprende totalmente al inicio de la fracción "cabeza", mientras que el furfural recién aparece al final de la fracción "cuerpo" y levanta sustantivamente en la fracción "cola". Los valores de la muestra fueron bastante bajos comparados con la NTP del pisco.

La acidez volátil, expresada en términos de ácido acético, en el pisco se refleja en forma creciente desde el inicio de la destilación de la fracción "cabeza" y sube significativamente en la parte final de la fracción "cuerpo" y parte de "cola" para luego descender. En la muestra analizada tuvo un valor despreciable.

El metanol, que aparece por degradación química enzaimática de compuestos pectínicos provenientes de los hollejos y pulpa de la uva durante la fermentación, está influido por la temperatura de destilación y su presencia se muestra ascendente desde la fracción "cabeza", aún más en el "cuerpo" y destila principalmente en la "cola". Este es el motivo fundamental por lo que se recomienda que la maceración - fermentación de las uvas, especialmente aromáticas, se haga sin la presencia de orujos o con maceración en hollejos por no más de 18 a 24 horas; 


\section{De la Evaluación Organoléptica y Estadística}

Un panel semientrenado de cuatro jueces evaluaron cualidades tales como: aspecto, color, olor y sabor por preferencia hedónica.

Realizar la fermentación del mosto sin hollejos, asi como controlar que la temperatura del mosto no excediera $\operatorname{los} 25,5^{\circ} \mathrm{C}$, sumado al reposo y la separación de las borras antes de la destilación, influyeron en los resultados físico - químicos y organolépticos que se reflejan en el puntaje promedio dado por los jueces $(8,187)$ a la muestra. El valor del compuesto alcohólico metanol es pequeño, comparado con los valores de NTP. Es un pisco límpido, brillante, incoloro, equilibrado en sus cualidades de olor y sabor, que recuerdan a la uva de origen y otras frutas; se considera como poco alcoholizado y libre de olores y sabores extraños. La comprobación de la muy buena calidad del producto se da a partir del cálculo de la desviación estándar de cada una de las cualidades organolépticas del pisco:

a) Aspecto, color y sabor, $\operatorname{con} \mathrm{S}=0,4330$

b) Olor, $\operatorname{con} \mathrm{S}=0,707$

\section{Planilla Resumen de Fichas de Calificación}

Cuadro $\mathrm{N}^{\circ} 03$ Evaluación organoléptica del pisco puro aromático Italia

\begin{tabular}{|l|c|cc|c|c|}
\hline CUALIDADES & $\begin{array}{c}\text { JUEZ } \\
\mathbf{1}\end{array}$ & $\begin{array}{c}\text { JUEZ } \\
\mathbf{2}\end{array}$ & $\begin{array}{c}\text { JUEZ } \\
\mathbf{3}\end{array}$ & $\begin{array}{c}\text { JUEZ } \\
\mathbf{4}\end{array}$ & $\begin{array}{c}\text { PROM. } \\
\text { de. } \\
\text { Cualidad } \\
\mathbf{x}\end{array}$ \\
\hline Aspecto & 9 & 9 & 9 & 9 & $\mathbf{8 , 7 5}$ \\
\hline Color & 8 & 8 & 9 & 8 & $\mathbf{8 , 2 5}$ \\
\hline Olor & 8 & 7 & 8 & 9 & $\mathbf{8 , 0 0}$ \\
\hline Sabor & 7 & 8 & 8 & 8 & $\mathbf{7 , 7 5}$ \\
\hline $\begin{array}{l}\text { Promedio de la } \\
\text { muestra }\end{array}$ & & & & & $\mathbf{8 , 1 8 7}$ \\
\hline
\end{tabular}

Fuente: Elaboración propia (2008).

\section{CONCLUSIONES}

1.La uva (Vitis vinifera $L$ ) variedad Italia que se trabajó mostró una madurez un poco avanzada expresada en los $26,4^{\circ} \mathrm{Brix}$ (sólidos solubles) y $14,6^{\circ} \mathrm{Be}$ (azúcares) y una acidez total de $5,2 \mathrm{~g} / \mathrm{L}$ en ácido tartárico, que se correspondieron con la cosecha y proceso tardíos : comienzos de mayo 2008. Se controló peso, Brix (SS), densidad, acidez total y $\mathrm{pH}$.

2.El proceso fermentativo se inició con la siembra de levaduras nativas del pie de cuba y se desarrolló durante 9 días, en ausencia de hollejos y en condiciones ambientales de clima frio, Se controló temperatura, no mayor de $25,5^{\circ} \mathrm{C}$ para evitar pérdida de aromas. También se controlaron Brix, densidad, acidez, $\mathrm{pH}$ y remontajes dos veces a dia .El vino base tuvo un rendimiento de $65,05 \%$ y un grado alcohólico de $11,10 \%$ vol. Se descubó y dio reposo por 24 horas para decantar y separar borras.
3.La destilación de 5,4 L de vino base se ejecutó en alambique de cobre, con fuego directo alto y luego moderado. Se separaron las fracciones de "cabeza" (1 \%), $54 \mathrm{ml}$ con 64,10\% vol. de grado alcohólico. Fracción "cuerpo" $(21,56 \%), 1100 \mathrm{ml}$ con 43,60\% vol. Fracción "cola" $(0,77 \%), 42 \mathrm{ml}$ y $9,80 \%$ vol. de grado alcohólico.

4.La evaluación química del destilado por cromatografia de gases demostró que el pisco tuvo todos sus valores conformes, según la NTP 211.001: 2006. Pisco Requisitos.

La evaluación organoléptica realizada por un panel de 4 jueces semientrenados que calificó utilizando una ficha de preferencia hedónica, otorgándole 8,187, equivalente a muy bueno, en una escala de 0 a 9 puntos.

El análisis estadístico por cálculo de la desviación estándar (S) sobre las calificaciones de los jueces muestra un valor de $\mathrm{S}=0,433$ para las cualidades de aspecto, color, sabor y de $\mathrm{S}=0,70$ para el olor, lo cual es satisfactorio.

\section{REFERENCIAS BIBLIOGRÁFICAS}

ALFÉREZ GARCÍA, Lourdes (2006). Efectos de la Temperatura, Contenido de Orujos y Fosfato de Amonio en la Fermentación de Mosto para Obtención de Pisco Puro Aromático Italia (Vitis vinífera $L$ ). Tesis para optar el titulo de Ingeniero en Industrias Alimentarias. FAIA/ UNJBG-Tacna, Perú.

CERRO RUIZ, Samuel (2007). Efectos del proceso de elaboración en la caracterización fisico-química yorganoléptica de un pisco puro de uva Italia (Vitis vinifera L).PROIN, FAIA/ UNJBG 2006 -Tacna, Perú.

HATTA SAKODA, B.(2004). Influencia de la fermentación con orujos en los componentes volátiles del pisco de uva Italia (Vitis vinifera L. var. Italia). Tesis de Maestría. Escuela de Postgrado. Universidad Nacional Agraria La Molina. 2004. Lima.

HUAQUISACA QUISPE, Óscar F. (2005) Estudio de la Influencia de la maceración y la temperatura en la obtención y caracterización del pisco puro aromático Italia (Vitis vinifera L. var. Italia). Tesis para optar el titulo de Ingeniero en Industrias Alimentarias. FAIA / UNJBG - Tacna, Perú.

INDECOPI - Norma Técnica Peruana 211.001 : 2006 Bebidas Alcohólicas. Pisco. Requisitos.

PALMA J.C. y SCHULER RAUCH J. (2004). "Evaluación del efecto de tres sistemas de destilación en la calidad del pisco de uva quebranta en el Perú -III Congreso Nacional del Pisco. 